\title{
Normal Pressure Hydrocephalus after Myodil Myelography
}

\author{
Ezgi Akar, Mehmet Ufuk Akmil
}

Haydarpaşa Numune Training and Research Hospital, Department of Neurosurgery, Istanbul, Turkey

\begin{tabular}{|c|c|}
\hline \multirow[t]{3}{*}{ Abstract } & $\begin{array}{l}\text { Objective: Fat-saturated contrast agent-enhanced myelography was used for } \\
\text { the purpose of diagnosing most spinal lesions from the beginning of the } 19^{\text {th }} \\
\text { century until the end of the } 20^{\text {th }} \text { century. Myodil was among the agents used } \\
\text { for many years for this purpose. }\end{array}$ \\
\hline & $\begin{array}{l}\text { Methods: A rare long-term complication of myodil myelography is presented } \\
\text { with a cerebrospinal fluid (CSF) absorption disorder occurring years after } \\
\text { myelography that caused a secondary normal pressure hydrocephalus. }\end{array}$ \\
\hline & $\begin{array}{l}\text { Results: A case of normal pressure hydrocephalus with a slowly developing } \\
\text { clinical picture that emerged years after a ventriculoperitoneal shunt for a } \\
\text { myodil myelography } 25 \text { years ago due to suspicion of lumbar spinal stenosis } \\
\text { was treated. }\end{array}$ \\
\hline $\begin{array}{l}\text { Received: } \\
\text { Jyly 23, } 2014\end{array}$ & $\begin{array}{l}\text { Conclusions: Many acute and chronic complications of fat-saturated } \\
\text { contrast agents were reported. Because of these complications and with } \\
\text { the technological developments in due course, these agents are replaced } \\
\text { by computerized tomography (CT), magnetic resonance imaging (MRI), or }\end{array}$ \\
\hline Revised: & \\
\hline November 6, 2014 & Keywords: Normal pressure hydrocephalus, myelography, myodil \\
\hline $\begin{array}{l}\text { Accepted: } \\
\text { February 9, } 2015\end{array}$ & IJIHS. 2015;3(1):39-43 \\
\hline
\end{tabular}

\section{Introduction}

Myodil (iophendilate, a fatty acid that was formerly manufactured by Glaxo Laboratories London; also known as Pantopaque in the USA) is an oil-based positive contrast media. ${ }^{1}$ The use of myodil in myelography was described for the first time in $1994 .^{2}$

Until the end of the $20^{\text {th }}$ century, Myodil was used for myelography, ventriculography, and cisternography. Complications caused by these oil-based contrast agents, ranging from intermediate transient reactions to severe meningeal reactions that could result in death, were reported. In addition to the complications that may occur during the early period after the application, some chronic complications may also be observed after many years. Those chronic complications are considered to be related to the arachnoiditis and disorders of cerebrospinal fluid (CSF) absorption

Correspondence:

Ezgi Akar, Haydarpaşa Numune Training and Research Hospital, Department of Neurosurgery, Istanbul, Turkey e-mail: ezgiaycicek@gmail.com caused by myodil. Some cases of obstructive hydrocephalus, thoracic arachnoiditis as well as syringomyelia in the late period after myodil myelography were described in literatures.,

In our case, normal pressure hydrocephalus (NPH) was developed secondary to the myelography using myodil performed 25 years ago.

\section{Case}

An 83-year old female patient was presented to our clinic with complaints of dizziness, balance impairment, amnesia, gait disturbance, and urinary incontinence. These complaints had lasted for one year and had been increasing over the last several months. In the physical examination, cranial examination was normal and the patient had no motor deficit. Deep tendon reflexes were normoactive and plantar skin reflex was flexor. The patient was walking slowly with short steps and a slowing of the psychomotoric functions was determined in mental tests. The followings were observed on the magnetic resonance imaging (MRI) result: 


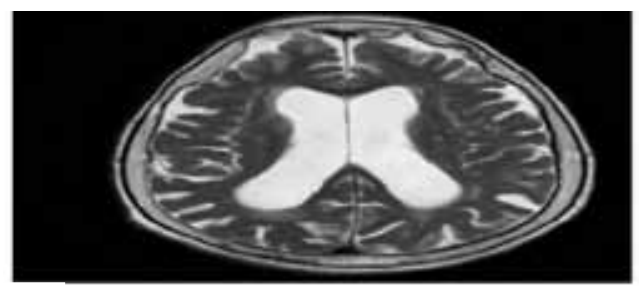

a

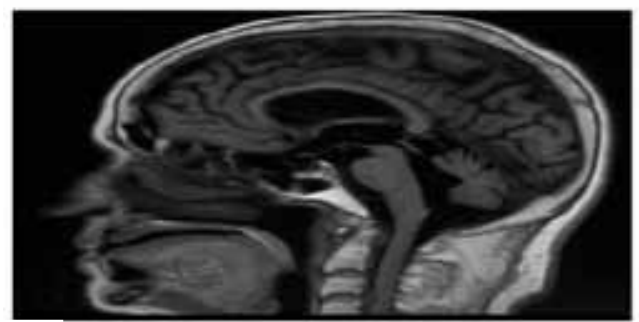

C

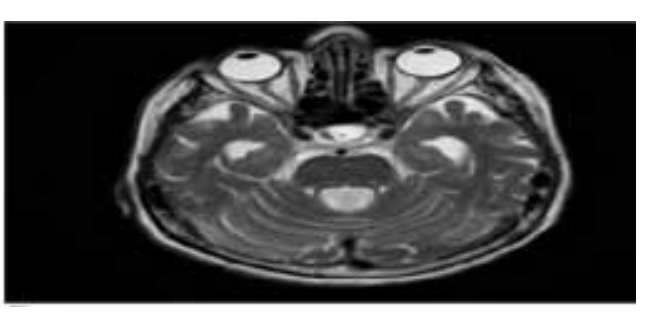

b

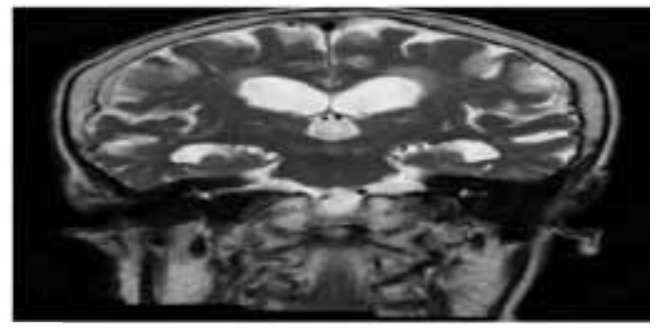

d

Fig. 1 Fig. 1a, b, c, d: Enlarged Temporal Horns are Seen on the Axial T2-weighted Images Increased Curvature of Corpus Callosum and Normal Size $4^{\text {th }}$ Ventricle are Seen on the Sagittal MRI Image Enlarged Lateral and $3^{\text {rd }}$ Ventricle are Seen on the Coronal MRI Image

increased lateral and third ventricle sizes with fourth ventricle size within the normal range; marked temporal horns; increased Evans index; enlarged sylvian fissures on coronal sections; and narrowed sulcus and subarachnoid space (Fig. 1a, b, c, d). Dynamic CSF MRI images showed the followings: a relatively decreased CSF flow; adequate flow at aqueduct level but with decreased output; and 98\% regurgitation. Cerebral and cerebellar atrophy were seen. A lumbar puncture (lumbar tap) test was performed for three days with 30 cc CSF extracted once a day. The CSF appeared to be clear and its pressure was $17 \mathrm{~cm} \mathrm{H}_{2} \mathrm{O}$. The walking speed of the patients increased after the lumbar tap test was conducted and a significant improvement was observed in the mental test. Shunt surgery was planned for the patient who benefited from the evacuative lumbar puncture. A gravity assisted medium pressure shunt was placed through the right Frazier's point. Significant improvement was seen in the symptoms of the patients after the surgery. Postoperative antibiotic therapy was initiated. Shunt catheter was seen in its place on the control computerized tomography (CT) image performed postoperatively (Fig. 2a). On the CT image, multiple millimeter- sized hyperdense oval lesions were seen in the cisterns and, especially, in the posterior fossa (Fig. 2b, c, d, e). During the retrospective MRI image inspections, myodil remnants were not observed (Fig. 3a, b, c). When another inquiry were made on the past medical history of the patient again, it was revealed that the patient had undergone a myodil myelography procedure 25 years ago due to the suspicion of lumbar spinal stenosis for back and leg complaints. The result of the myelography was normal and the patient had benefited from the conservative treatment. Hyperdense oval lesions seen on the CT images were myodil remnants. NPH developing in the patient years after the procedure is quite likely to be a late complication of myodil myelography. The patient was discharged on 4 days after the operation.

\section{Discussion}

Normal pressure hydrocephalus or NPH is a condition defined for the first time in 1965 by Adams and Hakim with dementia, impairment of balance, and urinary incontinence as the classic clinical finding triad. NPH has been 


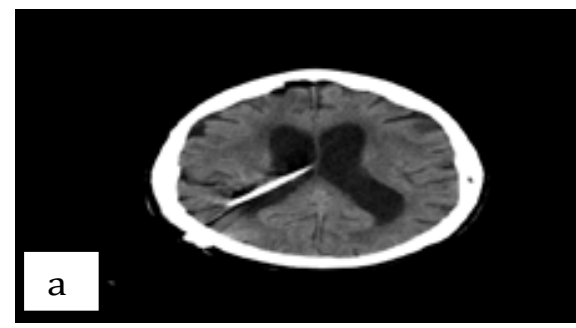

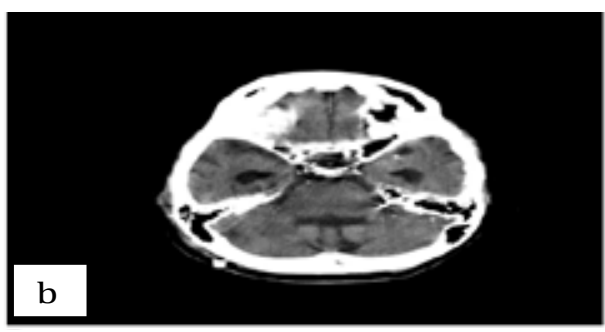

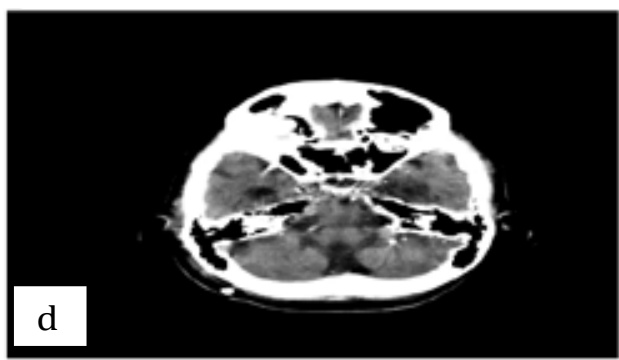

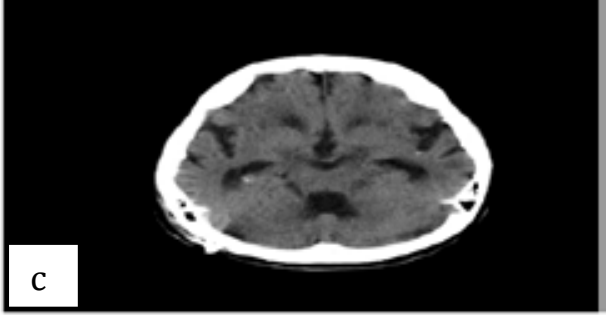

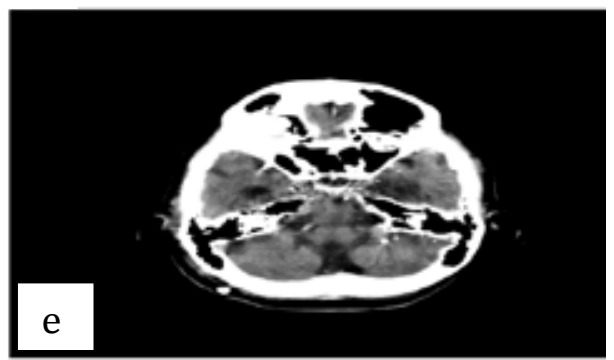

Fig. 2 Shunt Catheter was Seen on the Postoperative CT Image. Fig. 2b, c, d, e: Multiple Millimeter-sized Hyperdense Myodil Remnants were Observed in the Posterior Cisterns, Prepontine Cistern, Lateral Ventricles and Sylvian Fissures.

observed with declined cognitive functions, hence it is more frequently found in elderly patients. It is diagnosed based on the classic clinical findings, ventricular dilatation, and normal CSF pressure. ${ }^{5,6}$ It is usually seen as idiopathic NPH (iNPH) of unknown etiology. If it occurs due to acquired causes such as meningitis, $\mathrm{SAH}$, or congenital-developmental causes, it is called secondary NPH (sNPH) ${ }^{5,6}$ It is commonly manifested by nonspecific slow progressing symptoms in the elderly patients. Impairment of CSF flow due to idiopathic or secondary reasons causes hydrocephalus. ${ }^{5}$

The imaging methods used in the diagnosis include computerized tomography, MRI, CSF flow dynamic studies, and cisternography. ${ }^{5}$ Ventricular dilatation, Evans index (which is a ratio of the maximum width of the frontal horns to the maximum width of the inner table of the cranium) of more than 0.3 , and increased corpus callosum curvature on CT and MRI images support the hydrocephalus diagnosis. Coronal MRI sections specifically provide useful information regarding NPH. In coronal imaging, sylvian fissures and large sulci under sylvian fissures, narrowed sulci at the high cerebral convexity, and distribution of CSF disproportionately between superior and inferior brain surfaces in addition to ventriculomegaly support diagnosis of NPH. ${ }^{5}$

In the case where imaging findings were found together with one finding of clinical triad, tap test is recommended. A positive tap test shows that the patient can benefit from surgical treatment. The CSF removal test is divided into two types; small and large volume removal of CSF. Small volume $(30-50 \mathrm{~mL})$ and large volume $(300-500 \mathrm{~mL})$ of CSF removal are carried out via a lumbar tap. A tap test is less invasive. Removal of larger volumes of CSF 

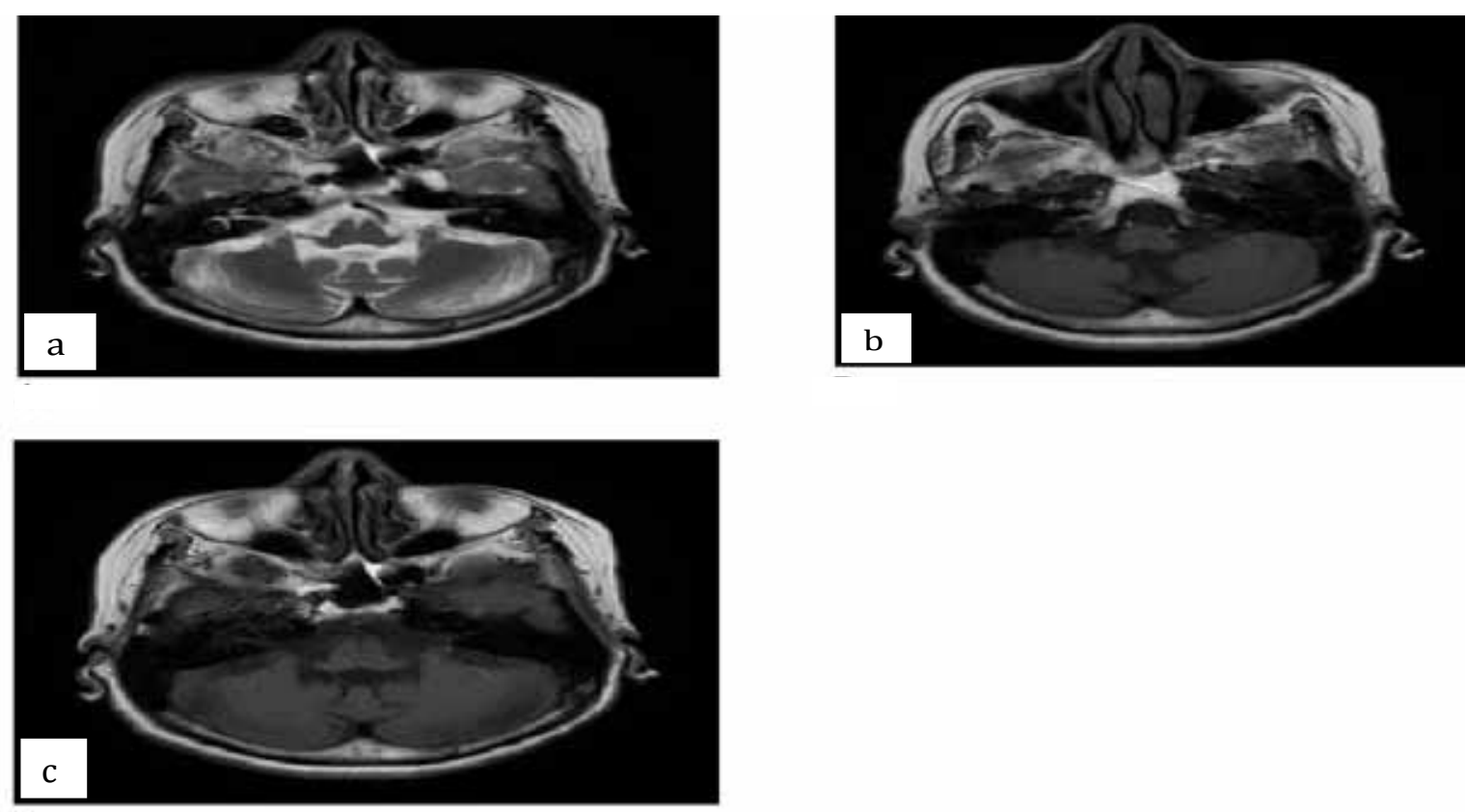

Fig. 3 Fig. 3a, b, c, Myodil Remnants were not Observed on the MRI Images

is carried out via external lumbar drainage for a few days. ${ }^{5,6}$ In the case where improvement in clinical symptoms after CSF removal was observed, tap test is considered to be positive. Improvement in daily life activities can be assessed with modified Rankin Scale (mRS). Gait can be assessed quantitatively using 3 meter timed up and go test or the 10 meter straight walk test. The mini-mental state examination, frontal assessment battery, and/ or trail making tests are applied for assessing cognition. ${ }^{5}$ CSF is colorless and clear and its pressure is maximum $18 \mathrm{~cm} \mathrm{H} \mathrm{H}_{2} \mathrm{O}-20 \mathrm{~cm} \mathrm{H}_{2} \mathrm{O}$. Shunt procedures are also applied for the other communicating hydrocephalus types in NPH. Most commonly, low or intermediate pressure ventriculoperitoneal shunts are preferred. ${ }^{5,7}$

A rare long-term complication of myodil myelography is presented in this case study. The Cerebrospinal fluid absorption disorder that occured years after myelography had caused secondary normal pressure hydrocephalus.

Myodil is an oil-based contrast agent that was manufactured and sold by Glaxo Laboratories in 1946 to 1988. After 1946, myodil was primarily used for myelography, ventriculography and cisternography. Myodil was sold in approximately fifty countries, including the United Kingdom. On September $19^{\text {th }} 1988$, Glaxo Laboratories declared that sales of myodil were ceased in United Kingdom in pursuance to the commercial decision of the Ministry of Health of the country. However, they still held the license for its production since it was still sold worldwide. ${ }^{1}$ The product is called as iophendilate (pantopaque) in USA. ${ }^{2}$

Myodil is seen as intradural droplets in the radiographic images. It is seen as hyperdense, oval, multiple droplets on the CT images. Due to its fat-like structure, myodil is seen as fat and fat-like structures on the MRI images which is hyperintense on the T1-weighted sequences and iso-hypointense on the T2-weighted sequences. ${ }^{1}$ Therefore, its radiological image has a lipoma, hemorrhage, hemangioma, and, in fact, melanoma like appearance. ${ }^{1}$

Complications caused by the use of myodil ranges from intermediate transient systemic reactions to severe meningeal reaction and death, which may occur immediately after myodil myelography. ${ }^{2}$ Acute complications may occur after use of myodil, while chronic complications may develop years after. ${ }^{1-4}$ This contrast media can be detected in the thecal sac even many years after myelography due to its remarkably slow absorption. ${ }^{1}$ Long- 
term complications seen after use of oilbased contrast agents like myodil are related to the development of arachnoiditis or CSF absorption disorders. The risk for developing arachnoiditis after using myodil is very low (1\%) and arachnoiditis can develop in the spinal or cranial regions. . $^{1,3,4,8,9}$

A normal pressure hydrocephalus condition had developed secondary to the myelography performed 25 years ago by using myodil in

\section{References}

1. Oo M, Wang Z, Sakakibara T, Kasai Y. Magnetic resonance imaging findings of remnants of an intradural oil-based contast agent: report of a case. J Spinal Cord Med. 2012;35(3):187-90.

2. Jensen F, Reske-Nielsen E, Ratjen E. Obstructive hydrocephalus following pantopaque myelography. Neuroradiology. 1979;18(3):139-44.

3. Gnanalingham KK, Joshi SM, Sabin I. Thoracic arachnoiditis, arachnoid cyst and syrinx formation secondary to myelography with Myodil, 30 years previously. Eur Spine J. 2006;15(5):661-3.

4. Kubota M, Shin M, Taniguchi M, Terao $T$, Nakauchi J, Takahashi H. Syringomyelia caused by intrathecal remnants of oil-based contrast medium. J Neurosurg Spine. 2008;8(2):169-73.

5. Mori E, Ishikawa M, Kato T, Kazui H, Miyake H, Miyajima M, et al. Guidelines for management of idiopathic normal pressure hydrocephalus: second edition. Neurol Med Chir (Tokyo). 2012;52(11):775-809.

6. Malm J, Graff-Radford NR, Ishikawa M, Kristensen B, Leinonen V, Mori E, et al. this case study. With time, CSF absorption was impaired gradually.

Complications of the oil-based contrast agents like myodil can still be encountered years after. With the lights of these acute and chronic complications of the contrast agents, contemporary CT, MRI and also water-based contrast agents like metrizamide (amipaque) and iohexol (omnipaque) are currently more frequently used. . $^{3,8-10}$
Influence of comorbidities in idiopathic normal pressure hydrocephalus- research and clinical care. A report of the ISHCSF task force on comorbidities in INPH. Fluids Barriers CNS. [serial on the internet]. 2013 June [cited 2014 April 12];10(22):[about 14 p.]. Available from: http://www.fluidsbarrierscns.com/ content/10/1/22.

7. Ishikawa $M$. Idiopathic normal pressure hydrocephalus--overview and pathogenesis. Brain Nerve. 2008;60(3):211-7.

8. Hugh AE. The subdural space of spine: A lympathic sink? Myodil' s last message. Clin Anat. 2010;23(7):829-39.

9. Gopalakrishan CV, Mishra A, Thomas B. Iophendylate myelography induced thoracic arachnoiditis, arachnoid cyst and syrinx, four decades later. Br J Neurosurg. 2010;24(6):7113.

10. Gupta SR, Naheedy MH, O’Hara RJ, Rubino FA. Hydrocephalus following iophendylate injection myelography with spontaneous resolution: case report and review. Comput Radiol. 1985;9(6):359-64. 